Relations industrielles

Industrial Relations

\title{
Le management d'aujourd'hui, Savoir organiser, Savoir décider, par Gérald Lefebvre, Montréal, Les Éditions de l'Homme, 1975, 166 p.
}

\section{Jean-Pierre Beaulieu}

Volume 30, numéro 4, 1975

URI : https://id.erudit.org/iderudit/028668ar

DOI : https://doi.org/10.7202/028668ar

Aller au sommaire du numéro

Éditeur(s)

Département des relations industrielles de l'Université Laval

ISSN

0034-379X (imprimé)

1703-8138 (numérique)

Découvrir la revue

Citer ce compte rendu

Beaulieu, J.-P. (1975). Compte rendu de [Le management d'aujourd'hui, Savoir organiser, Savoir décider, par Gérald Lefebvre, Montréal, Les Éditions de l'Homme, 1975, 166 p.] Relations industrielles / Industrial Relations, 30(4), 799-800. https://doi.org/10.7202/028668ar

Tous droits réservés (C) Département des relations industrielles de l'Université Laval, 1975
Ce document est protégé par la loi sur le droit d'auteur. L’utilisation des services d'Érudit (y compris la reproduction) est assujettie à sa politique d'utilisation que vous pouvez consulter en ligne.

https://apropos.erudit.org/fr/usagers/politique-dutilisation/ 
par le Conseil au chapitre 2, mettront tout en oeuvre pour bloquer la démarche vers le libre-échange parce qu'ils risquent de perdre le bien-être économique assuré artificiellement par le protectiunnisme. Tout au moins ils exigeront des compensations durant la période de transition: paiements d'assurance-chômage et autre assistance financière pour la main-d'oeuvre déplacée, subventions et allègements fiscaux pour les industries manufacturières devant disparaître, «restructurer leur production, développer de nouvelles lignes de produits et mettre sur pied de nouvelles facilités de commercialisation à l'étranger » (p. 186). Ces coûts ne seront pas négligeables, surtout en période de stagnation, et même si l'on peut escompter des bénéfices nets positifs d'une politique libre-échangiste, le rapport aurait dû leur accorder une attention plus importante que celle manifestée au chapitre 13. De plus il aurait intérêt à expliciter d'avantage la recommandation 18 qui paraît aux yeux des lecteurs des plus nébuleuses.

c) Qu'en est-il en terminant des effets régionaux d'une politique libreéchangiste canadienne? Le rapport du Conseil Economique est très explicite à ce sujet: «En régime de libre-échange unilatéral ou multilatéral, c'est au Québec d'abord, et en Ontario ensuite, que l'emploi diminuerait le plus, là encore parce que c'est dans cette dernière province que se concentrent les industries faisant concurrence aux importations, et qu'au Québec une forte proportion des industries ont un haut coefficient de main-d'oeuvre » (p. 186). Ces anticipations facilement prévisibles à cause des raisons citées ci-haut demandent donc des mesures spécifiques d'aide gouvernementale et des politiques d'encadrement pour faciliter le transfert des ressources. Or encore là, le rapport du Conseil pèche par optimisme. Il suggère l'implantation «de nouvelles industries ayant un avenir à long terme dans les régions menacées par la concurrence des importations » (p. 192); ceci demeure un voeu pieux surtout lorsqu'on regarde les résultats des programmes actuels, pourtant nombreux et bien pourvus. La même remarque s'applique à sa proposition d'une étroite collaboration fédérale-provinciale «pour assurer la cohésion de tous les aspects de l'aide canadienne à l'adaptation" (p. 193). Enfin, lorsqu'il avance l'idée que "ces diminutions de production et d'emploi se- raient toujours atténuées par toute dépréciation de la valeur du dollar canadien » (p. 185), il semble oublier un instant qu'une telle éventualité ferait augmenter le prix général des importations, résultat qui contrebalancerait un des effets majeurs d'une politique de libre-échange.

Nous avons répondu dans ce court texte au désir du Conseil Economique d'activer les discussions publiques autour d'une politique aussi importante pour l'avenir du Canada. Nos remarques sont demeurées pourtant partielles ne faisant pas ressortir les innombrables qualités et richesses d'informations contenues dans ce rapport. A ce titre, le document "Au-delà des frontières》 ainsi que les études spéciales qu'il résume, méritent amplement d'être lus.

\section{Carmine NAPPI}

Ecole des Hautes Etudes

Commerciales de Montréal

Le management d'aujourd'hui, Savoir organiser, Savoir décider, par Gérald Lefebvre, Montréal, Les Editions de l'Homme, 1975, $166 \mathrm{p}$.

Voici un volume à lire. A lire par les administrateurs, à lire par les étudiants concernés par le sujet et à lire par les théoriciens et professeurs pour y découvrir comment on peut rendre simples et opérationnelles diverses théories du management.

Dans la première partie, «Les notions de base », Lefebvre nous introduit à son conception de la gestion: "gérer, c'est gouverner ou veiller à ce que tout fonctionne pour le mieux, » (p. 20). "Pour gérer, il faut prévilégier certains éléments de la réalité au détriment des autres, il faut simplifier» (p. 22). On ne peut mieux illustrer le style simple et sans prétention de l'auteur. Derrière cette apparente simplicité se retrouve une profonde compréhension et intégration des sujets discutés qui en permettent une présentation claire et concrète.

Dans cette première partie, le chapitre trois, «L'organisation : un système ouvert », est une excellente synthèse et clarification de l'approche-système appliquée à l'organisation. Il est facile de sophistiquer cette approche de façon à éloigner plus d'un lecteur. Lefebvre fait exactement le contraire. Il manifeste un 
souci constant de demeurer en contact avec le lecteur et de mettre celui-ci en situation d'action par rapport à l'organisation dont il peut être membre: «Un système de gestion fondé sur cette conception (l'approche-système) favorise une plus grande capacité d'agir, c'està-dire, plus de pouvoir pour les humains...» (p. 48).

La seconde partie, «Le système de gestion par objectifs, » est vraiment la partie-clef de cet ouvrage. Cette partie est essentiellement opérationnelle.

Dans un premier chapitre, l'auteur traite de la mission d'une organisation, des objectifs et des rôles reliés aux objectifs. Par la suite, il explique dans des chapitres distincts les différentes étapes à suivre pour analyser des situations organisationnelles, déterminer des objectifs, établir un plan d'action et le contrôler. Deux autres chapitres complètent cette partie. L'un qui présente «Un processus pour intégrer les éléments de la gestion », l'autre traitant de "L'implantation d'un système de gestion par objectifs».

Il existe beaucoup d'écrits sur le sujet de la gestion par objectifs ( MBO »). Peu se révèlent aussi près des réalités de l'organisation et des préoccupations quotidiennes des gestionnaires. Lefebvre explique étape par étape, illustre à l'occasion par des exemples simples et concrets, présente des grilles d'analyse, toujours avec un style direct qui conduit à l'action.

La troisième partie, «Sur l'art de la gestion » fournit un chapitre intéressant sur le travail d'équipe. Aujourd'hui, beaucoup d'organisations et de gestionnaires ont la hantise du travail d'équipe et des comités. La lecture de ce chapitre permet de désamorcer ce sujet, de le situer dans un contexte approprié et d'apprendre quand et comment utiliser le travail d'équipe.

Somme toute un excellent ouvrage, écrit et publié au Québec par un praticien du développement organisationnel; à notre connaissance c'est une première qu'il convient de souligner.

\section{Jean-Pierre BEAULIEU}

Alcan, Arvida
Répertoire de décisions en application du Code du travail, tome II, Mesures disciplinaires, par J. G. Descôteaux, Ottawa, Editions de l'Université d'Ottawa, 1974, $268 \mathrm{p}$.

Ce deuxième et dernier tome du Répertoire du professeur Descôteaux comblera un besoin certain dans l'ellaboration de synthèses d'ordre pédagogique et dans la recherche de solutions pratiques portant sur les problèmes soulevés par le congédiement pour activités syndicales (C. du t., art. 14 et ss.). S'il est en effet assez facile de connaître les décisions des commissaires-enquêteurs et jugements du tribunal du travail publiés depuis 1969 , il en va autrement pour la période antérieure. Le premier mérite de l'ouvrage de Me Descôteaux est d'avoir rassemblé et résumé les décisions rendues entre janvier 1962 et 1969 par les organismes alors compétents. L'importance de plusieurs de ces décisions peut être contestée même de nos jours, ne fusse que d'un point de vue comparatif. Le second mérite, moins perceptible, consiste à avoir regroupé pour fin de classification sous des thèmes précis les décisions et jugements rendus de 1969 à fin 1973. Le professeur Descôteaux comble ainsi un vide manifeste. La multiplicité des décisions rendues depuis janvier 1969 rendait en effet laborieuse l'identification des espèces jurisprudentielles selon les divers sujets particuliers que soulèvent l'application et l'interprétation des dispositions du Code sur le congédiement pour activités syndicales.

Les divers cas jurisprudentiels répertoriés dans l'ouvrage ont été regroupés en neuf grandes catégories générales codifiées comme suit: 100- Généralités; 200- Compétence des organismes; 300Mesures disciplinaires pour activités syndicales: congédiement, suspension, non-rappel, déplacement, mise à pied; 400- Réintégration et indemnité; 500 Fardeau de la preuve; 600- Revision de la décision; 700- Délai de soumission de la plainte; 800- Infractions pénales; 900- Requête pour permission d'appeler. Ces catégories ont elles-mêmes été divisées en autant de sous-catégories que le nécessitent la nature et la portée du prcblème. A titre d'exemple, la catégorie "Réintégration et indemnité 》 (no. cod. 400) donne lieu aux regroupements suivants: 400-01- Réintégration; 400-02-Indemnité; 400-03- Période servant à déterminer l'indemnité; 400-04- 